\title{
Lo Andino y lo no Andino. Perspectivas arqueológicas comparativas entre las tierras bajas y las tierras altas
}

Peter Kaulicke ${ }^{1}$

\begin{abstract}
Resumen
Las etiquetas "andino" y, en menor grado, "amazónico" se caracterizan por simplificaciones esencialistas basadas en prejuicios políticos e indigenistas que se descubren como invenciones poco realistas y desprovistas de elementos de juicio sustentados, sobre todo en sus dimensiones históricas y prehistóricas. Desde perspectivas arqueológicas se presenta un panorama distinto complejo, dinámico, multidireccional en historias longevas de desarrollos independientes y de intercomunicaciones de diferentes intensidades y alcances. Por tanto, deberían superarse supuestas fronteras culturales o de otro tipo para llegar a una especie de megahistoria que cubriría buena parte de Sudamérica.
\end{abstract}

Palabras claves: Andinidad. Perspectivas comparativas. Arqueología, prehistoria. Andes. Amazonía.

\begin{abstract}
The tags "Andean" and "Amazonian" conceal essentialist simplifications caused by political and indigenist prejudices which are biased and unrealistic inventions devoid of sustained evidence. This is particularly true in the perspective of its historic and prehistoric dimensions. This perspective presents us with a different complex, dynamic, multidirectional background of long term histories of independent developments as well as intercommunications of varying intensities and scales. Therefore such illusionary frontiers should be demolished in order to build a kind a megahistory of almost pan-South American dimensions.
\end{abstract}

Keywords: Andean studies. Comparative perspectives. Archaeology. Prehistory. Andes. Amazonia.

"Lo andino", "andinidad", "hombre andino" o algo más institucionalacadémico "estudios andinos" (como Centro de Estudios Regionales Andinos Bartolomé de las Casas en Cuzco [desde 1974] e Instituto Francés de Estudios Andinos [desde 1964]) son términos usados a menudo entre antropólogos, sociólogos y arqueólogos para referirse a un ámbito geográfico que incluye los Andes de Ecuador, Perú y Bolivia y a sus habitantes amerindios. Se

1 Pontificia Universidad Católica del Perú, Lima. 
suelen postular vínculos culturales compartidos entre las comunidades contemporáneas sobre bases históricas y más aún, pre o protohistóricas compartidas. Sin ánimo de discutir la complejidad y problemática de sus múltiples definiciones y problemáticas es evidente que estas a menudo están afectadas por posiciones nacionalistas e indigenistas (véase Ricard [ed.] 2005). Tienden a cubrir las evidentes diferencias internas de la actualidad por elementos unificados bajo rubros como identidad y etnicidad esencialistas, lo que adquiere el sentido de cultura como bloque inamovible en tiempo y espacio. Con frecuencia, esta posición ahistórica e introspectiva se vincula con una supuesta unicidad que implica una esencial incomparabilidad que complica la necesaria definición de "lo no andino". Una posible diferenciación podría reducirse a elementos geográficos como los Andes o las tierras altas, pero los Andes septentrionales de Colombia y los del sur como Chile y Argentina al sur de Bolivia suelen considerarse "menos andinos". En el caso del Perú, la franja costeña pese a tratarse de tierras bajas, en cambio, está incluida tácitamente en lo andino. La Amazonia, pese a formar una parte importante del territorio moderno del Perú, como en los casos de los demás estados andinos, es notablemente "menos andino" y marginada como lo muestran los problemas político-económicos con los indígenas amazónicos del Perú en la actualidad. Cabe anotar también que los estudios antropológicos sobre las comunidades nativas tanto serranas como amazónicas en el Perú no destacan siempre por su calidad y cobertura. Esto vale, sobre todo, para los estudios de comunidades serranas que a menudo se reducen a lo que suele llamarse folklore entre los estudiosos peruanos. Las comunidades amazónicas se caracterizan por la presencia de muchas lenguas vivas a diferencia de un monolingüismo castellano o un bilingüismo con el quechua o en menor grado el aimara en los Andes Centrales. Como las primeras comparten muchos rasgos con las comunidades de Brasil los estudios respectivos se orientan hacia la antropología brasileña neoestructural de corte francés que ha desarrollado una sofisticación respetable en las últimas décadas.

\section{La Amazonía y los Andes: ¿una oposición milenaria?}

Si nos concentramos en situaciones pre- o protohistóricas, estas diferencias se hacen más patentes. El imperio de los incas, de los siglos XV y XVI, está tomado como modelo primigenio de la andinidad por tratarse de la única civilización alta de América del Sur con carácter unificador de buena parte de los Andes. Las sociedades amazónicas o de las tierras bajas al este de los Andes, en cambio, fueron consideradas de diferentes maneras, pero en general, de un nivel cultural y político notablemente inferior. Esto queda evidente en el mapa de las áreas culturales de Steward (Steward 1948). Los Andes Centrales aparecen como "native civilizations" y otra área inmensa hacia el este como 
"Tropical Forest", con otras palabras imperio/estado contrapuesto a tribus, solo poco por encima de los llamados "marginales". Esta clasificación geográficoevolucionista implica que solo en los Andes hubo una secuencia de cambios notables mientras que en otras áreas hubo un estancamiento. Mientras que los andinos logaron construir paisajes por medio de la agricultura, los amazónicos se quedaron en un nivel de la horticultura (roce y quema), suplementada con pesca y caza/recolecta. Por tanto, gracias a tecnologías avanzadas los pueblos andinos superaron los retos del medio ambiente mientras que los amazónicos quedaron a la merced del mismo. Este último argumento se basa en severas restricciones del medio ambiente que contradicen la exuberancia de la biodiversidad por lo que Betty Meggers lo ha llamado "counterfeit paradise" (paraíso fraudulento, Meggers 1971, 1996, 2007). Debido a ello cerámica compleja debería haberse introducido a la Amazonia por medio de los Andes orientales (Evans y Meggers 1968:108) debido a migración y difusión (ibid., figs.2:80). Donald Lathrap, el pionero de la arqueología amazónica en el Perú, en cambio, sostuvo la posición inversa (Lathrap 1970). Señaló que la mayor cantidad de las plantas domésticas se originan en las tierras bajas, tratándose en su mayoría de tubérculos, en particular la yuca (Manihot utilissima), no solamente por su relevancia alimenticia sino también por tratarse de la preferida bebida consumida en fiestas. Su argumento para la existencia de migraciones y difusiones es de orden lingüístico combinado con complejos cerámicos en un procedimiento que sigue usándose (véase Neves 2009). Según él, estas migraciones se inician en la Amazonía Central en dirección occidental a partir de un proto arawak y un proto tupi-guaraní (Lathrap 1970, fig.5). Con ello es la Amazonía que da origen a las civilizaciones andinas y no viceversa (véase abajo). Ya el arqueólogo peruano Julio C.Tello había planteado un origen similar concentrándose en los Andes Orientales a partir de la década de los veinte del siglo pasado (Tello 1929, 1942). Investigaciones arqueológicas más recientes y la ecología histórica han llevado a otro vuelco en la apreciación de la complejidad social de las sociedades amazónicas prehistóricas en el sentido de una densidad poblacional muy alta, la presencia de sitios habitacionales muy extensos con la presencia de formaciones políticas de notable sofisticación (véase Balée y Erickson 2006). Cabe señalar a modo de ejemplo que el sitio "capital" de Santarem, en la Amazonía Central, poco estudiado todavía,ocupaba un área nuclear con depósitos densos de aproximadamente 100 hectáreas y una extensión total de unos 25 kilómetros cuadrados comparado con ChanChan, la capital de Chimor en la costa peruana o Cahokia, el sitio más monumental de los Estados Unidos, los tres más o menos contemporáneos (Heckenberger 2008:952, Heckenberger y Neves 2009:256).

Estas contradicciones categóricas se deben probablemente a simplificaciones causadas por la generalización de relativamente pocos datos consolidados y sus combinaciones "cruzadas" de ambos lados (Andes y Amazonía) que provienen 
de la ecología con sus diferentes subdisciplinas, combinada o contrastada con antropología, etnohistoria, arqueología y lingüística. De ahí no puede sorprender la aparición de argumentos circulares que falsifican los postulados finales. Esta referida escasez de bancos de datos se debe entre otros factores a la dificultad de su obtención. La densa cobertura vegetal de la Amazonía reduce la visibilidad y, por tanto, contrasta notablemente con las condiciones áridas más favorables que caracterizan muchas partes de los Andes Centrales donde la detección de sitios arqueológicos resulta mucho más fácil. Solo recientemente se está aplicando técnicas más sofisticadas como el Airborne Laser Scanning que permite reconocer modificaciones de dimensiones reducidas en terrenos con coberturas vegetales densas (comunicación personal Prümers 2011). Otro problema están representado por la enorme extensión geográfica de la Amazonía que corresponde a poco menos del territorio de los Estados Unidos, mientras que los Andes Centrales ocuparían aproximadamente un tercio del mismo (véase mapa en Kurella/Neitzke 2002).

Estas y otras consideraciones que enfatizan las diferencias entre ambas áreas complican la posibilidad de perspectivas comparativas. Por tanto, conviene prestar más atención a la complejidad sincrónica y diacrónica y tomar en cuenta también los 500 años del impacto europeo y sus consecuencias para las poblaciones nativas.

\section{Los desarrollos culturales andinos y amazónicos}

Quisiera concentrarme en algunos de los aspectos claves para el desarrollo cultural tanto de los Andes como de las tierras bajas.

En primer lugar habría que contemplar el problema de la colonización inicial de América del Sur y, por tanto, de las poblaciones humanas más tempranas. Esta colonización parece haberse dado en el Pleistoceno Final pese a reclamos de presencia humana de 20.000 a 40.000 años o mucho más aún tanto para los Andes como para Brasil (véase Guidon y Delibrias 1986, Dillehay et al. 1992, Dillehay 2000:189-195) que carecen aún de evidencias convincentes aunque merecen estudios más detenidos. Por otro lado, no hay duda acerca de la presencia temprana en las tierras bajas y en un ambiente que hoy es la selva. Puntas foliáceas con pedúnculos del Holoceno temprano se parecen notablemente entre Colombia, Venezuela y Brasil (Meggers 2007, fig.4.9), aunque su distribución es más amplia (para el Perú véase Briceño 1999, Chauchat 1982, Maggard y Dillehay 2011, Kaulicke y Dillehay [eds.] 2013). Entre 14000 y 12000 años la presencia humana probablemente es aún esporádica, pero entre 10000 y 9000 se percibe un aumento notable. Esta presencia se da en ambientes muy diferentes que lleva a estrategias de subsistencia también variadas, la caza de megafauna es reducida, pero el uso de semillas en ambientes de bosques de Colombia, Brasil, Perú y Chile es común, por lo que Dillehay 
considera una amplia gama de recursos de subsistencia y la manipulación de plantas desde el Pleistoceno Final como "proto-Archaic foragers" a diferencia de una caza supuestamente especializada. Este punto es importante porque permite trazar cambios en la organización de sitios, la permanencia en ellos y la explotación y la manipulación de recursos en fechas muy tempranas. Estas tendencias a una especie de semi-sedentarismo se dan en muchos ambientes diferentes, tanto en áreas áridas como tropicales (Dillehay 2008).

Piperno (2011, fig. 14.1) distingue seis áreas donde la mayoría de las plantas domésticas pueden haberse originado, cuatro de ellas en o cerca de la cordillera andina, todas en diferentes tipos de bosques, en su mayoría bosques tropicales, una más en el área del Orinoco y otra en la parte tropical de Bolivia. Pese a ello, horticultores en sitios tempranos, como los del valle de Zaña en el norte del Perú cultivaron diferentes especies de varias de estas áreas en sus huertos a partir (y probablemente antes) de la Fase Las Pircas con horticultura establecida con el cultivo de zapallo (Cucurbita moschata), maní (Arachis hypogaea), yuca (Manihot sp.), posiblemente quinua (Chenopodium cf. quinua), frejoles (Phaseolus sp.) y pacae (Inga sp., un árbol frutal) (98007800 a.p.) (Rossen 2011). Pero en otras áreas otro tipo de recursos fue más importante como la pesca (véase Lavallée y Julien [dir.] 2012), la recolecta de caracoles terrestres y la obtención de fauna menor y mediana, en la costa norte del Perú (véase Chauchat et al. 1992) y quizá también en la Amazonía (para complejidad social incipiente véase Kaulicke 2012). Por lo general, esta fase temprana de la manipulación genética de plantas alimenticias no parece llevar directamente al proceso de la formación de las sociedades complejas y menos debe considerarse como factor causante de las mismas. Lo mismo es cierto para la cerámica que aparece en Colombia y Brasil con mucha anticipación a la más temprana de los Andes centrales, pero en contextos que tampoco implican la presencia de organizaciones sociales complejas (Roosevelt et al. 1991, para Colombia véase Oyuela-Caycedo y Bonzani 2005). Parece por tanto que aún en el Holoceno Temprano y más aún en el Holoceno Medio deben haber existido variados mecanismos de la distribución de rasgos económicos y técnicos sobre distancias notables, pero no sabemos aún cómo estas funcionaban debido a la escasez de evidencias concretas.

Se debe, por tanto, incluir otros aspectos más de orden cognitivo y ritual como formaciones de paisajes, interrelaciones entre paisajes funerarios, rituales y domésticos así como grados de cohesión y diferenciación sociales. En primer lugar podríamos contemplar la "monumentalización" del paisaje (véase Kaulicke 2001). Conchales aparecen muy temprano tanto en las costas del Perú, en el Ecuador, Colombia y Panamá como en las costas de Brasil (sambaquís) y Uruguay y hasta en los interiores como en la Amazonía Central. Muchos de ellos reflejan un marcado sedentarismo y, sobre todo en el caso de los sambaquís, y, más temprano en Las Vegas en el Ecuador (Stothert 1988), 
suelen ser monumentos de los muertos en cantidades asombrosas (Gaspar et al. 2008). Por tanto, son monumentos centrales y puntos esenciales de cohesión social.

Montículos de tierra tienen una distribución más amplia, pero aparecen muy temprano y, en casos excepcionales, siguen usándose en la actualidad, como entre los mapuche en el sur de Chile (Dillehay 2007). Este tipo a menudo aparece en grupos lo que permite correlacionarlos en complejos como reflejos de formas de organizaciones sociales (para un caso temprano en Uruguay véase Iriarte 2006, 2009, para el Brasil Neves 2009, Roosevelt 1991, Rostain 1999 [Upano, Ecuador] y entre otros). Como los conchales muestran diferentes ritmos de crecimiento y de funciones, pero, por lo general son productos sociales de larga duración. Es este aspecto que está adquiriendo más importancia en la arqueología de la Amazonía donde sirve para sugerir diferentes grados de complejidad social. A esto se agregan otros elementos como camellones (Denevan 2001) y sistemas de canalización, cuya distribución también es muy amplia si bien en forma de concentraciones desiguales en espacio y tiempo. Denevan (2001:304) compara los sistemas de cultivo entre la Amazonía y los Andes y concluye que hay similitudes pese a los diferentes biomas. Fuera de estos campos de cultivo hay evidencias de un mejoramiento de suelos y sofisticadas técnicas del manejo de recursos y modificaciones del paisaje. El cultivo intensivo y duradero permitía la presencia de poblaciones densas tanto en los Andes como en la Amazonía debido al mosaico de distribución desigual de recursos y factores sociales variables.

Pero el estudio de estas construcciones e instalaciones requiere excavaciones en área, documentación y análisis como base para interpretaciones más ambiciosas y con el fin de obtener datos más robustos para la elaboración de cronologías locales y regionales así como una base amplia de material arqueológico contextualizado. Estas podrían respaldar, modificar o rechazar modelos basados casi enteramente en reconstrucciones lingüísticas/etnohistóricas y "tradiciones cerámicas". Como ejemplo de un proyecto de larga duración se menciona aquí uno llevado a cabo por el Instituto Arqueológico Alemán en la región del Beni, en las tierras bajas de Bolivia. Se inició en 1994 y resultó en modificaciones de hipótesis reinantes sobre esta región arqueológicamente poco conocida pero muy importante (Prümers 2004). Entre otros avances destaca un contexto funerario "rico" en Loma Salvatierra, en un montículo, junto con otros más de 50 contextos. Estaba asociado con un disco de metal y orejeras metálicas, una tembetá de piedra, brazaletes de hueso, cadenas de dientes de felinos y restos de otros objetos orgánicos y corresponde al siglo VII d.C. Es el primer contexto de este tipo de diferenciación social en la Amazonía (Prümers 2009). Relacionados con el tratamiento de los muertos cabe señalar que la tradición chinchorro de la costa norte de Chile caracterizada por una manipulación extrema del 
cuerpo y una momificación artificial. En el lapso entre 3500 y 2000 a.p., está acompañada por la introducción de una serie de plantas alimenticias, entre ellas yuca y camote. Estas introducciones a la costa norte de Chile, basadas en análisis genéticos podrían deberse a la llegada de grupos humanos originarios de zonas tropicales orientales (Rothhammer et al. 2009).

De todo ello queda claro que no existía algo así como una especie de oposición cultural milenaria sino un mosaico complejo de desarrollos culturales tanto en las tierras bajas como las tierras altas dentro de redes de interacción de diferentes alcances espaciales y de intensidades en las largashistorias de sus existencias. Si bien el conocimiento de muchas áreas de los Andes centrales es mayor que el de la mayoría de las regiones de las tierras bajas, es preciso saber más sobre estas interacciones y sobre la organización de asentamientos, de paisajes, territorios y de la interacción entre muertos y vivos.

\section{Chavín y la Amazonía}

Para finalizar quiero presentar brevemente un caso célebre de vínculos culturales imaginados y algo más realistas entre la Amazonía y los Andes centrales en relación con el surgimiento del famoso fenómeno "chavín". Chavín de Huántar es un sitio en la sierra norte del Perú cuyas obras líticas decoradas en un estilo figurativo llamado "chavín" representan seres interpretados como pertenecientes al bestiario amazónico como caimán, anaconda, harpía, monos, etc., que obviamente no formaban parte de la fauna local o regional ni de la costa ni de la sierra (véase Burger 1995). Esta visión se debe mucho al mencionado arqueólogo Lathrap quien incorporó estos elementos con el fin de construir un origen amazónico de Chavín como también de los olmecas en Mesoamérica (Lathrap 1971, 1982, 1985). Además aparecen en este sitio y en otros con arte formativo de la esfera de interacción cupisnique (para mayores detalles, véase Kaulicke 2011, fig. 3) cabezas antropomorfas a menudo interpretadas como cabezas trofeo. Asimismo hay sugerencias de prácticas antropófagas y el consumo de drogas alucinógenas, todo en uso entre las etnias amazónicas recientes y subrecientes. La yuca y el cultivo de árboles frutales, también de origen de las tierras bajas, parecen haber tenido mayor importancia en las economías de subsistencia de estas sociedades costeñas y serranas. Tales prácticas, sin embargo, no están atestiguadas en las tierras bajas para el período en cuestión.. Si comparamos la extensión de la esfera de interacción cupisnique con sus características medioambientales vemos que se trata de selva alta que cubre la sierra y entra en las cabeceras de los ríos costeños (véase Kaulicke 2011). Se trata de un corredor natural entre la cuenca amazónica y la costa del Pacífico y constituye una frontera climática y topográfica. Esta zona es ecológicamente compleja y dominada por bosques de selva alta húmeda que alcanzan las cabeceras de los valles 
costeños. La componente faunística, por tanto, originalmente debe haber incluído animales como jaguar y otros felinos, boas y águilas con lo que esta fauna era bien conocida por las sociedades cupisniques. Estas condiciones medioambientales favorecen los vínculos entre la Amazonia y la costa y se expresan en la llamada esfera de interacción cupisnique que se extiende hasta Jaén y Bagua, donde a su vez parecen haber existido vínculos tempranos con el área de Chinchipe del Ecuador. Ahí, en Santa Ana- La Florida, se encontró un sitio excepcional con arquitectura monumental y contextos funerarios en los cuales destacan platos de piedra y ceramios (Valdez et al. 2008). Algunos de ellos llevan motivos muy parecidos a los más tempranos de la costa norte del Perú (en contextos acerámicos y cerámicos tempranos) (Valdez 2008) lo que corresponde también a la cronología de ambas zonas. Estos vínculos no se limitan a esta época temprana sino incluyen fenómenos como la incursión de material cultural afin al de la cerámica de los jívaros modernos que, entre 800 hasta 1200 DC se encuentra en el Chinchipe, Bagua, Jaén, Chachapoyas y el Huallaga (Guffroy 2006). Es muy probable también que las etnias coloniales como los guayacundos, ayabacas y clavas, así como los paltas y los malacatos en las tierras altas del Perú septentrional podrán corresponder a jívaros con lo que concuerda la toponimia (Hocquenghem 1989). Estas evidencias indican que podrían haber incursionando más al sur em tiempos anteriores. Un sitio temprano (Formativo Temprano hasta Horizonte Medio) en el depto. Cajamarca donde trabajé en la década de los setenta se llama Pandanche que indica la misma filiación jívaro que macanche (una boa de la costa de Lambayeque) (Kaulicke 2004, 2005). Por tanto, parece que existían tempranas redes de contacto entre los flancos tropicales de los Andes y la costa, aunque no necesariamente implican ni migraciones ni expansiones lingüísticas. Pero esta zona probablemente es de importancia central para el surgimiento de las sociedades complejas bien adaptadas a ambientes tropicales boscosos. En tiempos más tardíos estos vínculos probablemente se intensifican con una participación más activa de poblaciones de origen amazónico.

Volviendo a tiempos más tempranos debe recalcarse que supuestos rasgos "amazónicos" como aquellos mencionados en sierra y costa del Perú podrían rechazarse como intentos de comprobar supuestas raíces milenarias paralelas a aquellas que sustentan "lo andino". En todo caso queda por comprobar con evidencias contundentes que tales rasgos efectivamente son tan o más tempranos que en la costa norte del Perú lo que hasta ahora no se ha podido lograr aún.

\section{Conclusiones}

En vez de concentrarse en supuestas oposiciones basadas en identidades inventadas e intereses políticos actuales conviene concentrarse en historias 
de la longue durée en cuya construcción la participación de arqueólogos es indispensable. Estas historias dependen de cronologías consolidadas que permiten la elaboración de perspectivas comparativas más seguras. Lamentablemente este procedimiento, común en esta disciplina, está obstaculizado por el predominio de arqueologías nacionales o nacionalistas en América del Sur con tendencias excluyentes. Pero el problema que se presentó aquí requiere una perspectiva comparada más amplia que no solamente debería contemplar los vínculos entre los modernos países amazónicos o tierras bajas y sus historias compartidas sino también aquellos con los Andes no-amazónicos o tierras altas y otras áreas antes llamadas "marginales". No hay duda que las conexiones entre las tierras altas y bajas son longevas, dinámicas, fluctuantes y multidireccionales. Captarlas en toda su complejidad es una tarea que está por hacer en buena cuenta.

"Lo andino" no permite algo así como un "no-andino" ya que supuestamente es único e incomparable, pero esta supuesta unicidad ya implica una comparación. Tampoco permite una diferenciación interna ya que ésta relativizaría el constructo general. A la vez es de carácter ahistórico, por lo que constituye una generalización y valorización que encubre de un modo artificial complejidades dinámicas sociales existentes y por definir aún. En todo caso "lo amazónico" sería otro concepto algo artificial también que, como quería mostrarse en esta contribución no es una especie de oposición esencialista. Tanto las poblaciones "tradicionales" de la sierra como las de las tierras bajas se han formado en largos procesos históricos (desde la llegada de los europeos) y prehistóricos (con anterioridad a los últimos) que se deben entender como multitudes de historias con sus contingencias inherentes y entrelazadas que llevan a diferentes (micro)identidades en vez de una o varias mega-identidades artificiales.

\section{Referencias}

Balée, William y Clark Erickson. 2006. Time and Complexity in Historical Ecology: Studies in the Neotropical Lowlands. New York: Columbia University Press.

Briceño, Jesús. 1999. Quebrada Santa María: Las puntas de cola de pescado y la antigüedad del hombre en Sudamérica. En Peter Kaulicke (ed.). El Período Arcaico en el Perú: Hacia una definición de los orígenes, Boletín de Arqueología PUCP 3 (1998), pp.19-39.

Burger, Richard L. 1995. Chavin and the origins of Andean civilization. London: Thames and Hudson.

Chauchat, Claude; Elizabeth E. Wing, Jean-Paul Lacombe, Pierre-Yves Demars, Santiago Uceda y Carlos Deza. 1992. Préhistoire de la Côte nord du Pérou: le Paijanien de Cupisnique. Cahiers du Quaternaire 15. Bordeaux: Centre Régional de Publication de Bordeaux. 
Dillehay, Tom D. 2000. The Settlement of the Americas. A New Prehistory. Basic Books: New York.

Dillehay, Tom D. 2007. Monuments, Empires, and Resistance: Araucanian Ritual Narratives and Polity Formation, New York: Cambridge University Press.

Dillehay, Tom D. 2008. Profiles in Pleistocene History. En: Helaine Silverman y William W.Isbell (eds.). Handbook of South American Archaeology, pp.29-44, New York: Springer.

Dillehay, Tom D.; Gerardo Ardila Calderón, Gustavo Politis y María da Conceicao de Moraes Coutinho Beltrão.1992. Earliest Hunters and Gatherers of South America. fournal of World Prehistory 6,1, pp.145-204.

Denevan, William M. 2001. Cultivated Landscapes of Native Amazonia and the Andes, Oxford Environmental Studies, Oxford: Oxford University Press.

Evans, Clifford y Betty J. Meggers. 1968. Archaeological Investigations on the Río Napo, Eastern Ecuador. Washington, D.C.: Smithsonian Institution Press.

Gaspar, María Dulce, Paulo Deblasis, Suzanne K. Fish y Paul R.Fish. 2008. Sambaqui (Shell Mound) Societies of Coastal Brazil. En: Helaine Silverman y William W. Isbell (eds.). Handbook of South American Archaeology, pp. 319-335. New York: Springer.

Guffroy, Jean. 2006. El Horizonte corrugado: correlaciones estilísticas y culturales. Bulletin de l'Institut Français d'Etudes Andines 35 (3), pp. 347-359.

Guidon, Niède y Georgette Delibrias. 1986. Carbon-14 Dates Point to Man in the Americas 32,000 Years Ago. Nature 321, pp.769-771.

Heckenberger, Michael. 2008. Identity, Interaction, and Integration in the Tropical Forest. En: Helaine Silverman y William W. Isbell (eds.) Handbook of South American Archaeology, pp.941- 961, New York: Springer.

Heckenberger, Michael y Eduardo Góes Neves. 2009. Amazonian Archaeology. Annual Review of Anthropology 38, pp. 251-266.

Hocquenghem, Anne Marie. 1989. Los guayacundos de Caxas y la sierra piurana, siglos XV y XVI. Lima: CIPCA/IFEA.

Iriarte, José. 2006. Landscape Transformation, Mounded Villages and Adopted Cultigens: The Rise of Early Formative Communities in South-Eastern Uruguay. World Archaeology 38 (4), pp. 644-663.

Iriarte, José. 2009. La construcción social y transformación de las comunidades del Período Formativo Temprano del sureste del Uruguay. En: Peter Kaulicke y Tom D.Dillehay (eds.). Procesos y expresiones de poder, identidad y orden tempranos en Sudamérica, Segunda Parte, Boletín de Arqueología PUCP 11 (2007), pp. 143-166.

Kaulicke, Peter. 2001. Aree e tipología funerarie dell'America Meridionale. En: Antonio Giuliano (ed.). Enciclopedia Archeologica II, pp. 575-580. Roma: Istituto della Enciclopedia Italiana.

Kaulicke, Peter. 2004. Pandanche. En: Antonio Giuliano (ed.) Enciclopedia Archeologica Americhe Ocenia, pp.676-677. Roma: Istituto della Enciclopedia Italiana. 
Kaulicke, Peter. 2005 [1975]. Pandanche. Un caso del Formativo en los Andes de Cajamarca. Arqueología y Sociedad 16, pp.141-180.

Kaulicke, Peter. 2011. Algunas reflexiones sobre lenguas y sociedades en el Período Formativo centroandino. En: Peter Kaulicke, Rodolfo Cerrón-Palomino, Paul Heggarty y David Beresford-Jones (eds.). Lenguas y sociedades en el Antiguo Perú: Hacia un enfoque interdisciplinario. Boletín de Arqueología PUCP 14 (2010), pp. 123-139.

Kaulicke, Peter. 2012. On the Origins of Social Complexity in the Central Andes and Possible Linguistic Correlations. En: Paul Heggarty y David Beresford-Jones (eds.). Archaeology and Language in the Andes. A Cross-Disciplinary Exploration of Prehistory, pp. 111-133. Proceedings of the British Academy 173. Oxford: Oxford University Press.

Kaulicke, Peter y Tom D. Dillehay (eds.). 2013. Tradiciones andinas tempranas: cultura, tecnología y medioambiente. Boletín de Arqueológía PUCP 15 (2011).

Kurella, Doris y Dietmar Neitzke (eds.). 2002. AmazonasIndianer. LebensRäume LebensRituale LebensRechte, Berlin: Linden-Museum.

Lathrap, Donald W. 1970. The Upper Amazon, London: Thames and Hudson.

Lathrap, Donald W. 1971. The Tropical Forest and the Cultural Context of Chavín. En: Elizabeth P. Benson (ed.) Dumbarton Oaks Conference on Chavin. October $26^{\text {th }}$ and $27^{\text {th }}, 1968$, pp. 73-100. Washington, D.C.: Dumbarton Oaks Research Library and Collection.

Lathrap, Donald W. 1982. Complex iconographic features shared by Olmec and Chavín and some speculations on their possible significance. En: Jorge G. Marcos y Presley Norton (eds.). Primer Simposio de Correlaciones Antropológicas AndinoMesoamericano, 25-31 de julio de 1971, pp. 301-327. Guayaquil: Escuela Técnica de Arqueología.

Lathrap, Donald W. 1985. Jaws: The Control of Power in the Early Nuclear American Ceremonial Center. En: Christopher B. Donnan (ed.). Early Ceremonial Architecture in the Andes. A Conference at Dumbarton Oaks, $8^{\text {th }}$ to $10^{\text {th }}$ October 1982, pp. 241262. Washington, D.C.: Dumbarton Oaks Research Library and Collection.

Lavallée, Danièle y Michèle Julien (dirs.). Prehistoria de la costa extremo-sur del Perú. Los pescadores arcaicos de la quebrada de los burros (10000-7000 a.P.). Lima: IFEA/ Fondo Editorial PUCP.

Maggard, Greg y Tom D. Dillehay. 2011. El Palto Phase (13800-9800 BP). En: Tom D.Dillehay (ed.). From Foraging to Farming in the Andes. New Perspectives on Food Production and Social Organization, pp. 77-94. New York: Cambridge University Press.

Meggers, Betty.1971. Amazonia: Man and Culture in a Counterfeit Paradise. Chicago: Aldine.

Meggers, Betty. 1996. Amazonia: Man and Culture in a Counterfeit Paradise. Ed. Rev. Washington, D.C.: Smithsonian Institution Press. 
Meggers, Betty. 2007.Mid-Holocene climate and cultural dynamics in Brazil and the Guyanas. En: David G.Anderson, Kirk A. Maasch y Daniel Sandweiss (eds.) Climate Change \& Cultural Dynamics. A Global Perspective on Mid-Holocene Transitions, pp. 117-155. Amsterdam, Boston, Heidelberg, London, New York, Oxford, Paris, San Diego, San Francisco, Singapore, Sydney, Tokyo: Elsevier/Academic Press.

Neves, Eduardo G.. 2009. El Formativo que nunca terminó: La larga historia de estabilidad en las ocupaciones humanas de la Amazonía central. En: Peter Kaulicke y Tom D.Dillehay (eds.) Procesos y expresiones de poder, identidad y orden tempranos en Sudamérica, Segunda Parte, Boletín de Arqueología PUCP 11 (2007), pp.117-142.

Oyuela-Cayedo, Augusto y Renée M. Bonzani. 2005. San Juanito 1. A Historical Ecological Approach to an Archaic Site in Colombia. Tucsaloosa: The University of Alabama Press.

Piperno, Dolores. 2011. Northern Peruvian Early and Middle Preceramic Agriculture in Central and South American Contexts. En: Tom D. Dillehay (ed.). From Foraging to Farming in the Andes. New Perspectives on Food Production and Social Organization, pp. 275-284. New York: Cambridge University Press.

Prümers, Heiko. 2004. Hügel umgeben von "schönen Monstern“: Ausgrabungen in der Loma Mendoza, Bolivien). En: Expeditionen in vergessene Welten: 25 Jahre archäologische Forschungen in Amerika, Afrika und Asien, Forschungen zur Allgemeinen und Vergleichenden Archäologie 10, pp. 47-78.

Prümers, Heiko. 2009. ¿"Charlatanocracia” en Mojos? Investigaciones arqueológicas en la Loma Salvatierra, Beni, Bolivia. En Peter Kaulicke y Tom D. Dillehay (eds.). Procesos y expresiones de poder, identidad y orden tempranos en Sudamérica, Segunda Parte, Boletín de Arqueología PUCP 11(2007), pp.103-116.

Ricard Lanata, Xavier (ed.). 2005. Vigencia de "lo andino" en los albores del siglo XXI: una mirada desde el Perú y Bolivia. Cuzco: Centro de Estudios Regionales Andinos Bartolomé de las Casas.

Rossen, Jack. 2011. Las Pircas Phase (9800-7800 BP). En: Tom D. Dillehay (ed.). From Foraging to Farming in the Andes, pp. 95-115. New York: Cambridge University Press.

Rostain, S. 1999. Secuencia arqueológica en montículos del valle del Upano en la Amazonía ecuatoriana. Bulletín de l'Institut Français d'Etudes Andines 28 (1), pp. $1-37$.

Rothhammmer, Francisco; Calogero M. Santoro, Elie Poulin, Bernardo T. Arriaza, Mauricio Moraga y Vivien Standen. 2009. Archaeological and mtDNA evidence for tropical Lowland migrations during the Late Archaic/Formative in northern Chile. Revista Chilena de Historia Natural 82, pp.543-552.

Roosevelt, Anna. 1991. Moundbuilders of the Amazon: Geophysical Archaeology on Marajó Island, Brazil. San Diego: Academic Press.

Roosevelt, A,, R.A. Housley, M. Imazio da Silveira, S. Maranca y R. Johnson. 1991. Eight Millenium Pottery from a Prehistoric Shell Midden in the Brazilian Amazon. Science 254 (5038), pp. 1621-1624. 
Steward, Julian H. 1948. Culture Areas of the Tropical Forests. En: Julian H. Steward (ed.) Handbook of South American Indians 3, pp. 883-899. Washington, D.C.: Smithsonian Institution.

Stothert, Karen.1988. La Prehistoria Temprana de la Península de Santa Elena, Ecuador: Cultura Las Vegas, En: Miscelánea Antropológica Ecuatoriana 10, Guayaquil.

Tello, Julio C. 1929. Antiguo Perú. Primera Época. Lima.

Tello, Julio C. 1942 Origen y desarrollo de las civilizaciones prehispánicas andinas. En: Actas y Trabajos Científicos del $27^{\circ}$ Congreso Internacional de Americanistas, Lima 1939, pp.589-720. Lima.

Valdez, Francisco. 2008. Inter-zonal Relationships in Ecuador, en Helaine Silverman y William W. Isbell (eds.) Handbook of South American Archaeology, 865-887.New York: Springer.

Valdez, Francisco, Jean Guffroy, Geoffroy de Saulieu, Julio Hurtado y Alexandra Yépez. 2005. Découverte d'un site cérémoniel formatif sur le versant oriental des Andes. En: Palévol 4, pp. 369-374.

Data recebimento: $10 / 09 / 2012$

Data aceite: 27/05/2013 